\title{
Segmentation of high resolution remote sensing images by combining hidden Markov random field model and fuzzy c-means at the region level
}

\author{
Xu Song ${ }^{1,}$, Guoying Liu ${ }^{2, b}$ \\ ${ }^{1}$ Department of Computer and Information Engineering, Anyang Normal University, Anyang, China \\ ${ }^{2}$ Department of Computer and Information Engineering, Anyang Normal University, Anyang, China \\ asongxu518@126.com, bguoying.liu@aynu.edu.cn
}

Keywords: Image segmentation; Remote Sensing; Markov random field model; Fuzzy c-means; high resolution.

\begin{abstract}
In high spatial resolution remote-sensing images, complex landscapes are usually accompanied with macro texture patterns, which often adversely affect segmentation accuracy, mainly due to their high spatial and spectral heterogeneity. To address this problem, this study develops an image segmentation method by combining the iteration procedure of fuzzy c-means (FCM) clustering and hidden Markov random field (HMRF) model at the region level. The performance of the proposed method was assessed through aerial images. Results indicate that the proposed method can improve image segmentation accuracy, compared to FLICM, HMRF-FCM, MRR-MRF, and IRGS.
\end{abstract}

\section{Introduction}

Image segmentation is one of the most important steps in the interpretation of remote sensing images. It aims at partitioning an image into disjoint meaningfully homogeneous regions. In the past decades, many different image segmentation techniques have been proposed [1, 2, 3, 4]. Among them, those based on fuzzy c-means (FCM) [5] and Markov random field (MRF) models [6] are the most popular ones. Being able to retain more information from the original image, FCM has robust characteristics for ambiguity [7]. However, the standard FCM ignores the information about spatial context, which makes it very sensitive to noise, outliers, and other imaging artifacts. The MRF model provides a powerful and formal way to model the information about the mutual influences among image pixels. Nevertheless, it fails to consider image ambiguity because its estimation approach, such as expectation-maximization (EM), needs to regard pixel labels as known quantities [8,9].

Motivated by the success of region-level MRF-based segmentation methods, which make it possible to capture and utilize correlations over different image regions, in this study, we propose an image segmentation method by combining the iteration procedure of FCM and HMRF at the region level.

\section{The Proposed Image Segmentation Algorithm}

\subsection{Framework of the proposed algorithm}

Fig. 1 shows the image segmentation framework of the proposed RFCMHMRF algorithm. First of all, a deliberate over-segmentation of the original high spatial resolution image is obtained. Then, attributes for each region, such as its area and boundary length, are calculated, and also a region adjacency graph (RAG) is built according to the relationships between neighboring regions: Each region corresponds to a node of the RAG, and the relationship between two different regions is defined by their adjacency as edges of the graph. Finally, segmentation result is obtained by using an iterative procedure between one-step FCM and one-step HMRF. The spatial relationships between neighboring regions, as well as their attributes, are integrated into the prior probability of HMRF and 
the dissimilarity of FCM to capture and utilize the large range variations of macro texture patterns in the original image.

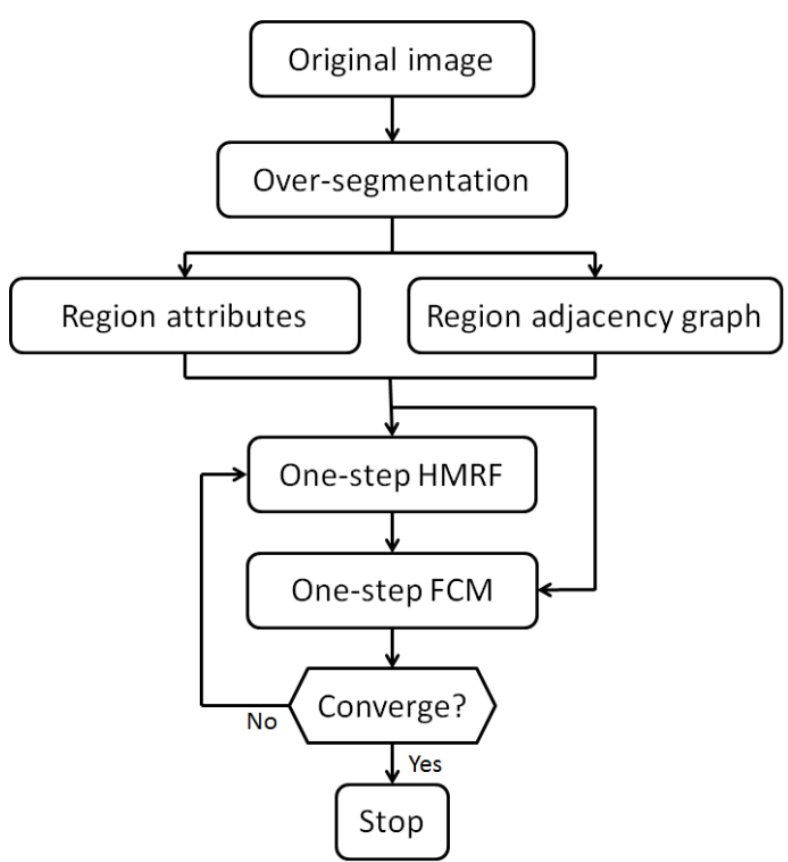

Fig. 1 The framework of the proposed method

\subsection{The core of the algorithm}

The main procedure of the proposed RFCMHMRF algorithm is as follows.

Step 1. Preparation: Set the number of different land-cover types ${ }^{K}$, the threshold of segmentation error ${ }^{\epsilon}$, and the maximal iteration number ${ }^{T}$. Get a deliberate over-segmentation, and then compute the boundary length ${ }^{b_{i, j}}$ and pixel number $n_{i}$ for each region.

Step 2. Initialization: In the first iteration $\left(t^{t=1}\right.$ ), derive the pixel-level fuzzy memberships by using the original FCM, and compute the initial region-level fuzzy memberships by

$u_{i, k}^{(t)}={ }^{\sum_{s \in r_{i}} u_{s, k} / n_{i}}$, then, establish the membership matrix $\left\{u_{i, k}^{(t)}\right\}$ and derive a segmentation $x^{(t)}$ by Equation (13).

Step 3. One-step HMRF: Derive the mean $\mu_{k}^{(t)}$ and covariance matrix ${ }_{k}^{\Sigma_{k}^{(t)}}$ for HMRF by Equation (11) and Equation (12), respectively. For each region, get the prior probability $P\left(x_{r_{\tilde{i}}}=k \mid \eta_{r_{i}}\right)$ by Equation (3) and the conditional probability $P\left(y_{r_{i}} \mid x_{r_{i}}\right)$ by Equation (5), and then generate the normalized joint probability matrix $\left\{z_{i_{2}, k}\right\}$ by Equation (7).

Step 4. One-step FCM: For each region, compute the dissimilarity function that is given by Equation (9), and then generate the distance matrix $\left\{d_{i_{\ell} k}\right\}$. Generate the new region-level fuzzy membership function that is given by Equation (10), and obtain the new membership matrix $\left.{ }^{\left\{i_{2}, k\right.}\right\}$, which is to be used in the next iteration process.

Step 5. Convergence: Derive a new segmentation result $x^{(t+1)}$ according to Equation (13). In case of convergence (the rate of pixel labels changed between two successive iterations is smaller than $E$ or ${ }^{t>T}$ ), stop iteration, output $x^{t}$; otherwise, set ${ }^{t=t+1}$, and go to Step 3 . 


\section{Experimental results and discussions}

In this section, to test the performance of the RFCMHMRF algorithm, we carry out experiments on aerial images. Apart from our algorithm, four competitive methods are used to compare the segmentation results:

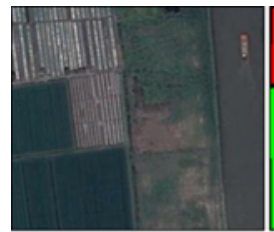

$(a)$

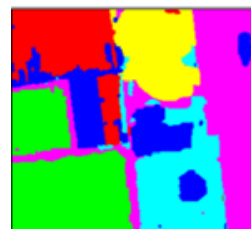

(e)

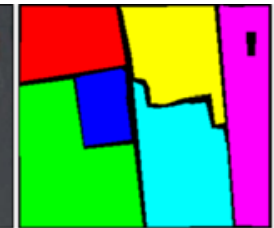

(b)

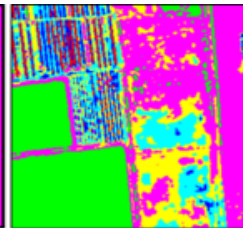

(c)

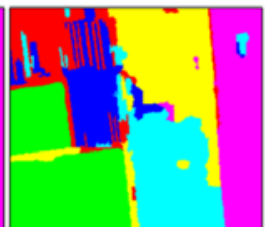

$(f)$

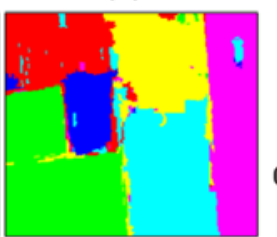

$(g)$

Agric. Land-I

$\square$ Grassland

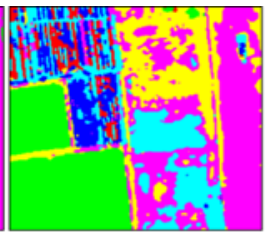

$(d)$

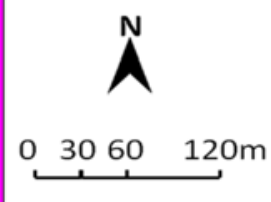

$\square$ Agric. Land-III

River

Fig. 2: Segmentation results of AER-SH. (a) AER-SH. (b) Ground truth. (c) Result of FLICM. (d) Result of HMRF-FCM. (e) Result of MRR-MRF. (f) Result of IRGS. (g) Result of RFCMHMRF.

FLICM [7]: It improves the traditional FCM by using fuzzy local (both spatial and spectral) similarity measure, aiming to guarantee noise insensitiveness and image detail preservation.

HMRF-FCM [8]: It combines fuzzy clustering and HMRF together to explore the spatial coherency modeling capabilities of MRF in the fuzzy clustering procedure.

MRR-MRF [11]: It uses the multiresolution region-based weighted average and difference to provide a good pixel-level descriptor for the large range variations of macro texture patterns.

IRGS [10]: It is a region-level MRF-based method, which uses region growing technique in searching for the solutions of the objective functions.

The overall accuracy ${ }^{o}$ and the Kappa coefficient ${ }^{\kappa}$ are used to measure the degree of similarity between segmentation results and the ground truth. Let $c_{i j}$ be the proportion of pixels that were assigned to the $i^{\text {th }}$ land-cover type of the segmentation result and $i^{\text {th }}$ land-cover type of the ground truth. Denote $c_{i-v}=\sum_{j=1}^{K} c_{i j}$ and $c_{-j}=\sum_{i=1}^{K} P_{i j}$, then ${ }^{o}=\sum_{i=1}^{K} c_{i i}$ and $K=\frac{\sum_{i=1}^{K} c_{i i}-\sum_{i=1}^{K} c_{i} c_{i}}{1-\sum_{i=1}^{K} c_{i} \cdot c_{i i}}$

Fig. 2(a), called AER-SH, is a piece of aerial image of an outskirt of Shanghai, China. Its size is $258 \times 271$ pixels. It includes grassland, bare soil, a river, and three types of agricultural land. Different types of macro texture patterns are distributed throughout the image. The deliberate over-segmentation is obtained by the meanshift algorithm [18] with the minimum area ${ }^{a=20}$, the spatial bandwidth $\rho=4$, and the range bandwidth ${ }^{r=4}$.

Table 1: Comparison of segmentation results.

\begin{tabular}{|l|l|l|l|l||}
\hline \multirow{2}{*}{ Method } & \multicolumn{2}{l|}{ AER-SH } & SP5-PS & \\
\cline { 2 - 5 } & $o$ & $k$ & $o$ & $k$ \\
\hline FLICM & 0.5443 & 0.4358 & 0.6294 & 0.4408 \\
\hline \hline HMRF-FCM & 0.6909 & 0.619 & 0.6909 & 0.5334 \\
\hline MRR-MRF & 0.8733 & 0.8298 & 0.8655 & 0.817 \\
\hline \hline IRGS & 0.8937 & 0.8698 & 0.8734 & 0.8069 \\
\hline \hline RFCMHMRF & 0.9524 & 0.9414 & 0.9086 & 0.8607 \\
\hline
\end{tabular}


Table 1 shows the overall accuracy and Kappa coefficient of each algorithm on AER-SH. Both indicators of the proposed algorithm have the highest values, which prove that the proposed method can largely improve the results of this scenario.

\section{Summary}

To address the problems caused by macro texture patterns in high resolution remote sensing images, in this study, we develop the RFCMHMRF algorithm by combining the iterative procedure of HMRF and FCM at the region level. With the region-level spatial and structural information modeled by the fuzzy prior probability function of HMRF and the probabilistic dissimilarity function of FCM, the negative impact of macro texture patterns in some complex land-cover types is reduced. Experimental results show that RFCMHMRF can obtain more accurate segmentation results than FLICM, HMRF-FCM, MRR-MRF, and IRGS, when segmenting images with macro texture patterns.

\section{Acknowledgements}

This work was supported by the National Natural Science Foundation of China under Grant 41001251, the International Technology Cooperation Project of Henan Technology Department of China, the Key Technology Projects of Henan Province of China under 13201210212, The Key Technology Projects of Henan Educational Department of China under Grant 13A520011, and the excellent Youth Teacher of Henan Educational Department of China (2011).

\section{References}

[1] Hung, C., S. Kulkarni, and B. Kuo, A new weighted fuzzy c-means clustering algorithm for remotely sensed image classification [J], IEEE Journal of Selected Topics in Signal Processing (2011): $543-553$

[2] Tuia, D., J. M. Mari, and G. C. Valls, Remote sensing image segmentation by active queries [J], Pattern Recognition (2012): 2180 - 2192

[3] Sziranyi, T. and M. Shadaydeh, Segmentation of remote sensing images using similarity-measurebased fusion-MRF Model [J], IEEE Geoscience and Remote Sensing Letters (2014): 1544 - 1548

[4] Zhang, L., K. Jia, X. Li, Q. Yuan, and X. Zhao, Multiscale segmentation approach for object-based land-cover classification using high-resolution imagery[J], Remote Sensing Letters (2014): $73-82$

[5] Bezdejk, J.C., Pattern Recognition with Fuzzy Objective Function Algorithms [D], Plenum Press, New York, 1981

[6] Besag, J. On the statistical analysis of dirty pictures [J], Journal of the Royal Statistical Society: Series B (1986): 259 - 302

[7] Krinidis, S. and V. Chatzis. 2010, A robust fuzzy local information c-means clustering algorithm[J], IEEE Transactions on Image Processing (2010): 1328 - 1337

[8] Chatzis, S. P.and T. A. Varvarigou, A fuzzy clustering approach toward hidden Markov random field models for enhanced spatially constrained image segmentation [J], IEEE Transactions on Fuzzy Systems (2008): 1351 - 1361

[9] Deng, H. and D. A. Clausi, Unsupervised image segmentation using a simple MRF model with a new implementation scheme [J], Pattern Recognition (2004): 2323 - 2335 
[10] Yu, Q. and D. A. Clausi, IRGS: Image Segmentation Using Edge Penalties and Region Growing [J], IEEE Transactions on Pattern Analysis and Machine Intelligence (2008): 2126 - 2139

[11] Zheng, C., L. Wang, R. Chen, and X. Chen, Image segmentation using multiregion-resolution MRF model [J], IEEE Geoscience and Remote Sensing Letters (2013): 816 - 820 